\title{
Teaching physics novices at university: A case for stronger scaffolding
}

\author{
Christine Lindstrøm and Manjula D. Sharma \\ School of Physics, University of Sydney, New South Wales 2006, Australia
}

(Received 11 October 2010; published 3 June 2011)

\begin{abstract}
In 2006 a new type of tutorial, called Map Meeting, was successfully trialled with novice first year physics students at the University of Sydney, Australia. Subsequently, in first semester 2007 a large-scale experiment was carried out with 262 students who were allocated either to the strongly scaffolding Map Meetings or to the less scaffolding Workshop Tutorials, which have been run at the University of Sydney since 1995. In this paper we describe what makes Map Meetings more scaffolding than Workshop Tutorials-where the level of scaffolding represents the main difference between the two tutorial types. Using a mixed methods approach to triangulate results, we compare the success of the two with respect to both student tutorial preference and examination performance. In summary, Map Meetings had a higher retention rate and received more positive feedback from students_-students liked the strongly scaffolding environment and felt that it better helped them understand physics. A comparison of final examination performances of students who had attended at least 10 out of 12 tutorials revealed that only $11 \%$ of Map Meeting students received less than 30 out of 90 marks compared to $21 \%$ of Workshop Tutorial students, whereas there were no differences amongst high-achieving students. Map Meetings was therefore particularly successful in helping low-achieving novices learn physics.
\end{abstract}

DOI: 10.1103/PhysRevSTPER.7.010109

PACS numbers: 01.40.Fk, 01.40.gb, 01.50.F-

\section{INTRODUCTION}

There is an ongoing debate about student-centered versus teacher-centered learning, and the closely related minimally guided learning versus direct instruction [1,2]. However, in recent times, some shared understandings have emerged [3-5] and the focus of the debate is shifting to meeting the needs and expectations of the learner. That the above-mentioned methods are both relevant features of a learning environment is acknowledged; it is the balance that needs researching $[6,7]$. This paper adds to the literature by investigating variables that influence the balance, with a particular focus on the level of student expertise. We also consider details of students' cognition to be important elements, as has been done by other physics education researchers $[8,9]$.

First year physics students have varied experience with physics prior to attending university, and they have different reasons for studying physics. This study examines one course that is designed for large numbers of students without high school physics.

The goal of this study was to investigate the effect of two different tutorial environments with different levels of teacher instruction on students' experiences with and performances in their first university physics course. The two tutorial types are Workshop Tutorials, which were

Published by the American Physical Society under the terms of the Creative Commons Attribution 3.0 License. Further distribution of this work must maintain attribution to the author(s) and the published article's title, journal citation, and DOI. introduced at this Australian research intensive university in 1995, and the more recently developed Map Meetings.

\section{THEORETICAL PHILOSOPHICAL PERSPECTIVE}

As researchers, we have interests in the teaching and learning of physics, and as educators, we have some opportunity to be creative and to sustain good teaching and learning practices within the constraints of, and embedded in, the culture of a research-focused department. The research process has been conceptualized using the framework described by Crotty [10]. We have constructed meanings from our engagement with our teaching and learning contexts reflecting our epistemological framework-what we study and what we learn emerge as partners in the generation of meaning. The theoretical perspective of our study is postpositivism, whereby we gain insights into the experiences of our student community using quantitative and quantitative data. Our methodology is survey-based research, and the setting is naturalistic with variables being explored and described within categories that naturally emerge from the data, rather than prestructured and predetermined categories.

\section{BACKGROUND}

\section{A. From novice to expert}

The theory known as model of domain learning attempts to explain the journey from novice to expert in academic domains by considering the interplay of prior knowledge, interest, and strategic processing [11]. The nature of each of these three factors changes markedly as the learner 
develops from novice, via competent learner, to expert, as do the optimal instructional methods. In particular, novices require careful scaffolding [11-15], which refers to the guidance of experts (or more knowledgeable peers) in the academic development of novices.

"[Scaffolding] connotes a custom-made support for the 'construction' of new skills, a support that can be easily disassembled when no longer needed. It also connotes a structure that allows for the accomplishment of some goal that would otherwise be either unattainable or quite cumbersome to complete." [16]

At some stage, however, scaffolding that is beneficial to novices becomes a hindrance to further learning for more competent students. This observation is referred to as the expertise reversal effect [15]. In tertiary physics it is unknown at what level of competency one instructional method becomes preferable over another.

Novices are characterized by their limited domain knowledge, which, in turn, can affect interest and strategic processing. Whereas experts generally have a strong internal interest in their subject, owing to their extensive domain knowledge, novices place a higher demand on the learning environment to maintain their interest [17]. Instructional methods that can maintain interest include adequate pacing of a lecture, reducing assumption about prior knowledge, explicitly flagging important concepts, connections, or results, providing challenges that are at a suitable level of difficulty, teaching students relevant learning strategies, teaching how to attack problems, and directing students to resources relevant for exam study $[17,18]$.

Strategic processing refers to how students learn new material [11]. Whereas most expert strategies rely heavily on prior knowledge for activities such as generating an initial overview, critical interpretation, and evaluation of content $[17,19,20]$, the limited knowledge of novices may prevent them from applying such strategies [21]. One consequence of the reduced availability of many strategies to novices is that novices require instructional methods that help them form overviews of content and be explicitly told how to approach material $[12,22]$, which is of particular importance to our study.

\section{B. Cognition and construction of meaning}

Level of knowledge and strategic processing are both features of cognition. The following briefly discusses the literature that elaborates on this. A key element of thinking occurs in working memory, which has very limited capacity and represents the bottleneck of cognitive processing [23]. The limitation refers to how many "chunks" can be held in working memory at any one time, where a chunk represents some integrated piece or unit of information [23]. However, a chunk can have considerable structure depending on prior knowledge [24]. Strongly associated chunks make up schemata or mental models, which describe the organization of knowledge structures held in long-term memory [24].

Cognitive load refers to the load imposed on working memory by a given task; it exists in three different forms: intrinsic, extraneous, and germane [15,25]. Intrinsic load is a property of the subject matter itself. It refers to the number of chunks that needs to be processed simultaneously to foster understanding and thus cannot be reduced by instructional design without reducing the quality of understanding. However, by initially introducing a subsection of a topic, the learner can integrate the initial elements into a coherent whole (one chunk) first, thereby freeing up space to allow for the integration of more elements. Extraneous load refers to tasks that are not conducive to learning. An example is a poorly written problem where the solver needs to use a part of his working memory to understand the problem in the first place. Extraneous load can be reduced by employing good instructional design. Lastly, germane load is the load placed on working memory in constructing schemata and developing automatization by introducing more structure in each chunk.

Well-developed schemata are important because schema activation not only depends on the existence of the particular structure in long-term memory, it also depends on how strongly and easily that knowledge is activated. Thus, the intrinsic load is a necessary but not sufficient condition for learning; it simply refers to the holding of several chunks of knowledge in working memory simultaneously. The germane load, on the other hand, is imposed by the process of integrating this knowledge into long-term memory. The situation in which the total load exceeds the capacity of working memory is referred to as cognitive overload. When designing instructional methods, it is important to consider the interplay between the various factors that place demands on students' working memories. Scaffolds designed in this study are intended to optimally facilitate strategic processing and meaning-making by managing cognitive load.

Time is another important factor in the construction of meaning; the process of transferring information to longterm memory can take hours or even days [23]. It is assumed that during this time of consolidation the construction of meaning is particularly vulnerable, and memories can easily be forgotten or disrupted [26]. This highlights the importance of revisiting material to ensure appropriate encoding in long-term memory, and supports interaction with material in different ways to give students views of the same material from different angles.

\section{Concept maps and knowledge maps}

Concept maps is probably the best known term used to describe a visual overview of several individual concepts and their relationships. Such maps are used as teaching aids, learning tools, and for assessment or evaluation $[6,21,27,28]$. They sometimes follow rules of construction, 
such as being read from top to bottom, and moving from general to more specific concepts as one descends down the map. The term knowledge maps is sometimes used when there is a set of nodes with verbal information interconnected with differentially nameable links [29]. Research has shown that knowledge maps used as learning tools enhance retention of main ideas within a domain [30] and are useful reference tools in problem solving [29]. In a study by Patterson, Danserau, and Newbern [29], psychology students learned about the effect of alcohol or cocaine on the human body. Students taught using knowledge maps as communication aids outperformed conventionally taught students, as measured by a combination of free recall and fill-in-the-blanks tests. The result was believed to be due to the clear overview such a map provided, as well as the reduced verbal content.

According to Kilic [31], the research on concept maps is more widespread in subjects such as biology [32-34] and chemistry $[35,36]$ than in physics. In physics, the majority of research into concept or knowledge maps uses these as assessment tools [28] (see, for example, [37-39]). This study focuses on Link Maps, visual maps specifically designed for physics [40]. These will be described later.

\section{Work in tertiary physics}

It is fairly common to have large numbers of students studying first year university science and mathematics courses as mandatory subjects in a range of degree programs such as health and environmental studies. Such courses are referred to as service subjects and for the Australian physics context a 2005 national study found that "Not surprisingly, given the role of service subjects in providing foundations early in a degree program the majority of first year offerings are service subjects" [41]. In general, many students studying service subjects are physics novices as they have not taken senior high school physics, the group we are examining in this project. As in most other subjects, research strongly indicates that the traditional lecture style is ineffective and alternative teaching methods are advocated $[42,43]$. In particular, interactive methods have provided significant gains in conceptual understanding [44], but these are generally not easily implemented [45-47]. The two different tutorials examined in this paper may be considered practical interactive instructional methods - one of which includes a visual aid specifically developed for physics-that aim to support student learning by carefully considering students' cognitive processes and interest (according to the points outlined in Sec. III A).

\section{TUTORIAL ENVIRONMENTS}

The School of Physics at the research-focused University of Sydney offers weekly 50-min tutorials to all its first year students. Fifty to 60 students are centrally allocated to each tutorial class. In 2007, a $2 \%$ mark was given for attending a minimum of 10 out of 12 tutorials, and students were allocated to either Workshop Tutorials or Map Meetings.

\section{A. Workshop Tutorials}

Workshops Tutorials are student- centered tutorials in which students are encouraged to work collaboratively in groups of four on problems provided in the tutorial. Such cooperation in which "relative novices work together to solve challenging learning tasks that neither could do on their own prior to the collaborative engagement" is known as peer collaboration [48]. The problem sheet contains qualitative, quantitative, and demonstration problems, where the latter is associated with simple experiments available in the tutorial room. Students answer problems on a team sheet, which is handed in at the end of the tutorial; this is not marked or returned. Three tutors allocated to each tutorial guide students and primarily respond to student questions. The tutors are instructed to interact Socratically with the students, a feature of such tutorials that has been found particularly beneficial for conceptual learning by other research groups as well [49]. See Sharma et al. [50] and Sharma et al. [51] for more details; a brief summary is provided in Table I.

Evaluations of Workshop Tutorials found that students with high school physics who attended more than half the tutorials performed statistically significantly better in the final examination than those who attended fewer than half the tutorials, even though the backgrounds of the two groups were not statistically significantly different (as measured by their exposure to high school physics and mathematics, and the "Tertiary Entrance Rank," an overall measure of academic performance in the last year of high school) [50]. Similarly, for students without high school physics, higher attendance correlated with higher examination mark [51]. However, interestingly, Sharma et al. [51] found that students who stayed with the same collaborative group throughout the semester performed significantly better than those who did not. Qualitative feedback indicated that students liked the relaxed atmosphere of the Workshop Tutorials, but some students suggested that a tutor summarize the tutorial content at the end of the tutorial [50]. Still, Workshop Tutorials are well established and the positive effects they have on student learning and the student experience have been researched and documented. Table I details the main differences between Workshop Tutorials and Map Meetings.

\section{B. Map Meetings}

Successfully developed and trialled in 2006 [40], the primary aim of Map Meetings is to help students with no prior formal physics instruction gain a better understanding of physics. These tutorials have student- and teachercentered activities in approximately equal amounts, and 
TABLE I. Comparing the two different tutorial environments.

\begin{tabular}{|c|c|c|}
\hline & Workshop Tutorials & Map Meetings \\
\hline Material available & $\begin{array}{l}\text { Problem sheet. Solution sheet provided at } \\
\text { the end of the tutorial. }\end{array}$ & $\begin{array}{l}\text { Link Map (in color). Problem sheet. Solution } \\
\text { sheet provided at the end of the tutorial. }\end{array}$ \\
\hline Structure & $\begin{array}{l}\text { Students work in groups of four on prob- } \\
\text { lems for } 50 \mathrm{~min} \text {. Tutors available to help. }\end{array}$ & $\begin{array}{l}10-15 \text { min summary lecture. } 25-30 \text { min group } \\
\text { work on problems in groups of four. } 5-10 \text { min } \\
\text { of supervisor going though a problem on the } \\
\text { board with the class. }\end{array}$ \\
\hline Level of scaffolding & Low. & Relatively high. \\
\hline $\begin{array}{l}\text { Demonstrations } \\
\text { (small experiments) }\end{array}$ & $\begin{array}{l}2-5 \text { different demonstrations available in } \\
\text { the room, which students can work with } \\
\text { when they choose. }\end{array}$ & $\begin{array}{l}\text { One demonstration if considered suitable (most } \\
\text { tutorials, but not all), which students can work } \\
\text { with when they choose during the problem- } \\
\text { solving session. }\end{array}$ \\
\hline Topic content & \multicolumn{2}{|c|}{ The same topic is covered in both tutorials. } \\
\hline Staff level & Three tutors per class. & Two tutors per class. \\
\hline Class size & \multicolumn{2}{|c|}{ 50-60 students are assigned to each tutorial class. } \\
\hline
\end{tabular}

are therefore considered more scaffolding than Workshop Tutorials.

In the first 10-15 min of Map Meetings, the tutorial supervisor discusses the weekly topic in a "summary lecture," which is centered around a Link Map (see Fig. 1) - a visual map that contains the key information covered (see Lindstrøm and Sharma [40] for details). Using an overhead projector, the tutorial supervisor introduces and discusses the information on the map section by section by "constructing" it using 4-6 transparencies that each contains a part of the map. This process is referred to as "layering." The students are given a color copy of the map and a problem sheet, and are in the following 25-30 min devoted to collaborative small group work with the supervisor and a tutor available for Socratic dialogue, similar to Workshop Tutorials. Qualitative, quantitative, and generally one demonstration problem, of varied complexity, are included on the problem sheet. In the final 5-10 min the supervisor discusses a difficult question and problem-solving strategies on the board with the whole class. Table I shows a short summary of the tutorial content. Lindstrøm and Sharma [40] provide further details on

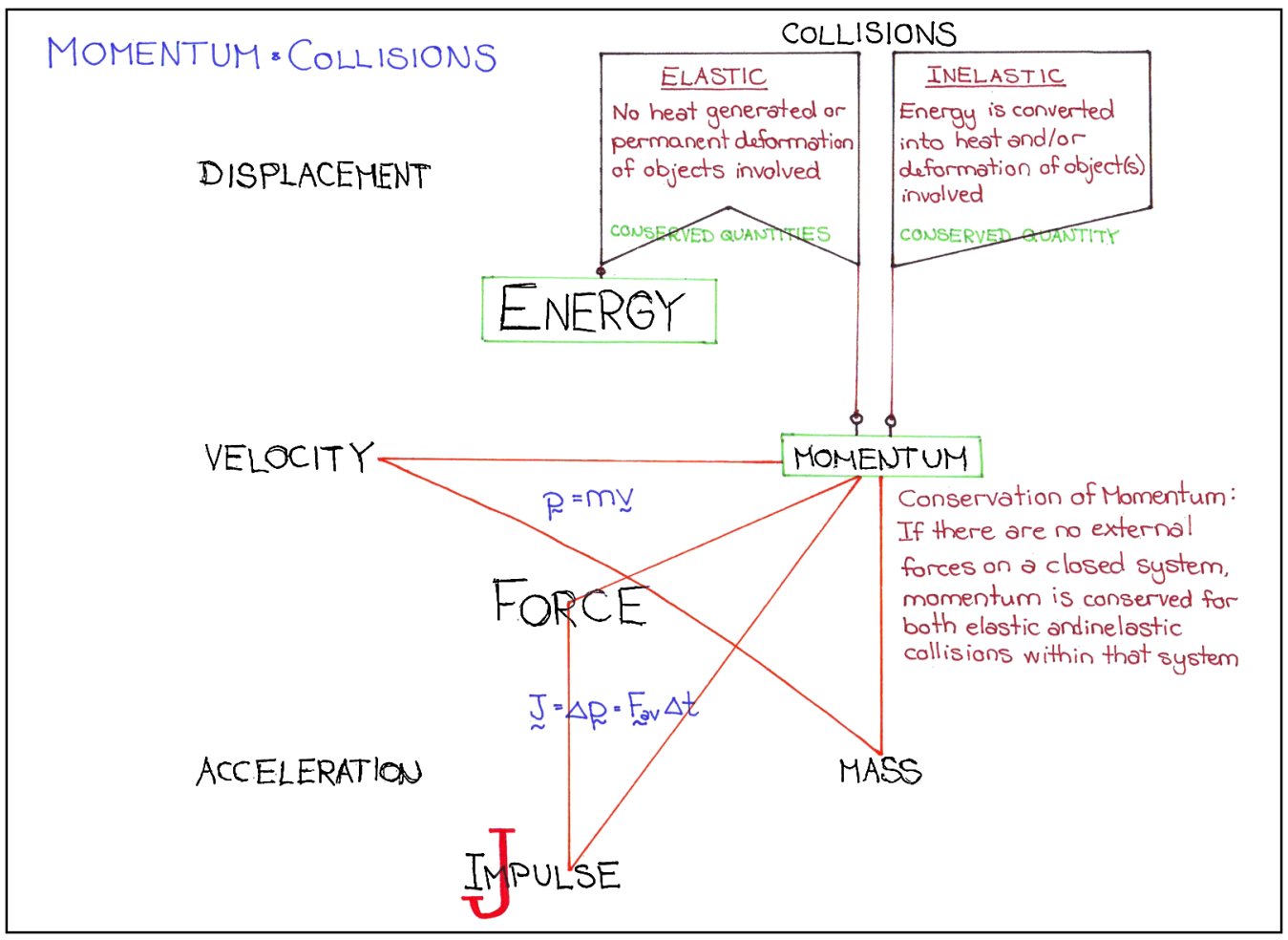

FIG. 1 (color online). Momentum and collisions map for the Fundamentals class. 
Map Meetings; here we will focus on how Map Meetings help scaffold student learning.

Based on knowledge of memory consolidation, within one week of initial exposure to new material, most students have not committed the new knowledge to long-term memory because this takes time and repeated exposure [26,52]. Revisiting important material therefore serves an important role in consolidating knowledge by increasing the probability of appropriate encoding into long-term memory. This was a key rationale for beginning Map Meetings with a summary lecture.

All Link Maps were produced by the first author rather than the students. This was done to capture an expert's interconnected view of the knowledge, the result of a lengthy, amorphous, and ongoing process as one develops from novice to expert. This choice was supported by research on physics novices, which has found that lowachieving students, in particular, are often unable to understand material at a deeper level without explicit instruction [12-14]. For example, in a study of preservice physics teachers, Ingec [39] found that although the participants had knowledge about the topic of impulse and momentum, they had difficulty establishing relationships among the concepts in a self-constructed concept map.

The specific design features of Link Maps were informed by research on knowledge structures and cognitive load theory. Link Maps make explicit the repeated use of the same fundamental concepts across different topics. By making explicit key links between concepts, extraneous load was expected to be reduced, assisting students to construct these important connections themselves, thereby increasing the capacity for germane load, which promotes learning. Note that although the connections were explicitly shown, students still needed to cognitively make these connections, a process requiring germane load. Temporal consistency is another feature aimed at reducing cognitive load through familiarity with the presentation style. Examples include placement of concepts, and colors used to represent concepts, symbols, units, equations, and definitions. Although the temporal consistencies provided a framework within which to structure the maps, the final layout of each map was a product of these general rules and what was considered to be the best representation of the given topic (see Fig. 1 for example of a Link Map).

In terms of how students are introduced to the Link Map, the method of layering explicitly directs students' attention to the concepts and their links. According to the "zoomlens" model of Eriksen and St. James [53], visual attention resembles a spotlight, where only the visual field within the spotlight focus is seen clearly. This way, intrinsic and extraneous cognitive load are reduced by drawing students' attention to concise and coherent subsections of the final map, which generally contain a considerable amount of detail.
The problem sheets were carefully designed with clear questions that focus only on a few chunks, without any unnecessary detail. The problems developed in complexity to scaffold understanding as task difficulty should be neither too hard nor too easy to promote learning $[18,54]$.

For the final board-solving session students would have already attempted the problem, as students are more likely to learn if they have worked on the material themselves first. Studies have shown that learning is most effective when explanations given are direct answers to student questions [55] or after students have worked on a difficult task and realized some of its differentiating features without necessarily having successfully completed it [56]. Teaching structured problem-solving strategies has also been found to support the development of conceptual understanding and foster a conceptual approach to problem-solving physics novices [57], and explicit teaching of metacognitive strategies has been particularly beneficial both for low-achieving students in secondary school [22] and first year university physics students [58].

\section{CONTEXT OF THE STUDY}

The Fundamentals course is a first semester first year course designed for students without prior formal physics instruction. The course aims to rapidly acquaint students with physics terminology so that they are able to undertake courses in second semester together with students who studied physics at high school.

At the University of Sydney, each semester lasts for 17 weeks: 13 teaching weeks, one midsemester break, and one nonteaching week before the final two examination weeks. Each teaching week has three one-hour lectures, one onehour tutorial, and one three-hour laboratory session. In addition, a duty-tutor is available for consultation two hours per day. Interactive teaching methods are embedded in lecturers, including buzz sessions (where students talk amongst themselves for a short period of time about a physics problem), interactive lecture demonstrations [59], and personal response systems [60], and questions from students are encouraged during lectures.

Course assessment is by assignments (10\%), tutorial attendance (2\%), laboratory work (20\%), an in-lab test $(8 \%)$, and a final three-hour examination $(60 \%)$. The examination was written by the lecturers who taught the course, which did not include the first author, who was the primary leader of the Map Meetings. The examination has 12 questions in total; the first six questions (five marks each) are conceptual while the remaining six (10 marks each) are traditional questions requiring both calculations and interpretation of answers. There are no multiple choice questions.

Figure 2 shows two representative examination questions. The first question (question 4) is a conceptual question concerning momentum and collisions. A successful response requires the student to explain the physical 


\section{Question 4}

Jack is in trouble! When he stood on the table at home, the table supported his weight. When Jack jumped from a ledge onto the same table, it broke.

(a) Draw the forces acting on Jack when he stood on the table. Do these forces represent an action-reaction pair? Explain your answer.

(b) Why did the table break when Jack jumped onto it? Explain your answer in terms of momentum, impulse and the forces acting when Jack was in contact with the table.

(c) After breaking one table, Jack found that he could safely jump onto a similar table if he placed a spongy mattress on top of it. Again, in terms of momentum, impulse and the forces acting, explain why this is possible.

(5 marks)

\section{Question 10}

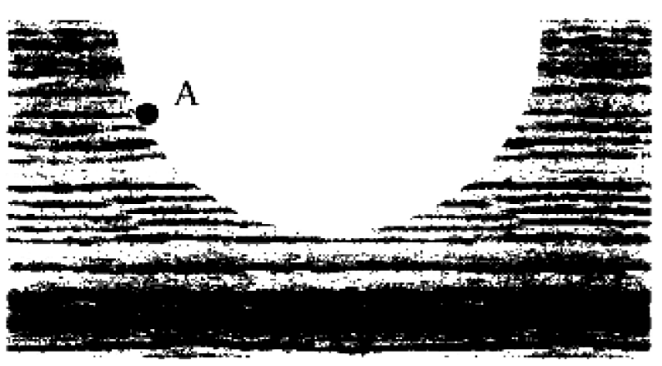

Cylindrical Ramp

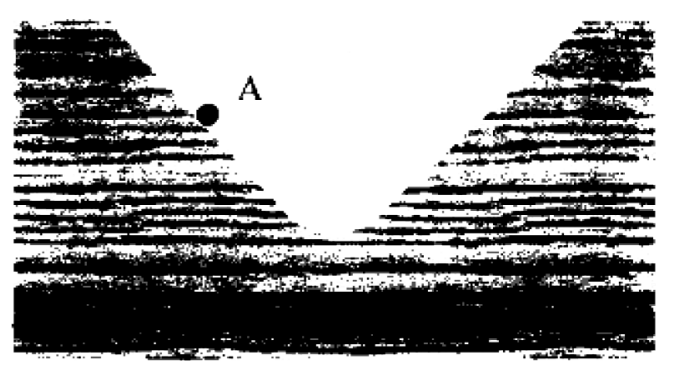

Wedge-shaped Ramp

Katie is a $55 \mathrm{~kg}$ skate boarder. She has a speed of $1.8 \mathrm{~m} \cdot \mathrm{s}^{-1}$ at a certain point $A$ on a cylindrical skateboard ramp, that is, one shaped as in the left-hand picture above.

Some time later Katie is moving with a speed of $6.0 \mathrm{~m} . \mathrm{s}^{-1}$ at some other point $\mathrm{B}$.

(a) By how much has her kinetic energy changed?

(b) Given that $265 \mathrm{~J}$ of mechanical energy is lost because of frictional forces in moving from A to B, has Katie moved uphill or downhill? Justify your answer.

(c) Find the change in her vertical position.

(d) If the ramp were wedge-shaped as in the right-hand picture above, but the height change was the same and the energy lost to frictional forces the same, would the final speed be the same? Justify your answer.

(10 marks)

FIG. 2. Two of the 12 questions in the examination paper. Question 4 is a conceptual question, whereas question 10 is a more traditional question.

situations using basic concepts from mechanics; the question cannot be solved with a plug-and-chug approach. Question 10 is a more traditional question, concerning energy. Some calculations are necessary, but of the four parts two require explanations demonstrating adequate understanding of the concepts and mathematics used.

Consequently, only about $50 \%$ of the marks are awarded for more traditional, mathematically based problem solving; for the remaining marks the students must display conceptual understanding of the physics explaining "real" situations. We therefore argue that the physics examination is a good measure of learning, striking a balance between conceptual and more traditional problems.

In this study, our goal was to investigate which tutorial type, Workshop Tutorials or Map Meetings, was more successful in engaging physics novices with physics learning in their first semester at university. A mixed methods approach was taken-collecting both qualitative and 
quantitative data - to allow triangulation of results [61] to help us form a detailed and in-depth understanding of the impact of the two types of tutorials on the student experience.

The specific research questions were (1) What are the patterns of student attendance? (2) How does an expert in physics education perceive these tutorial environments? (3) What are the students' experiences? (4) Is there a difference between students' examination performances, focusing, in particular, on low-achieving students?

\section{RESULTS}

\section{A. Sample}

The initial sample comprises all 262 students who attended the course, including those who did not take the final examination. Students were allocated tutorial classes centrally. Of five tutorial classes in total, we randomly chose two to be Map Meetings and the remaining three to be Workshop Tutorials. The first author was the supervisor in the two Map Meetings, whereas the Workshop Tutorials had three different supervisors. The initial attendance at Map Meetings and Workshop Tutorials was 107 and 151, respectively; four students never attended a single tutorial.

\section{B. Comparison of student backgrounds between tutorial types}

Since centrally allocating students to tutorial classes does not ensure randomness, students' backgrounds were compared. The end of high school aggregate mark, the Universities Admissions Index (UAI), was used as a measure of students' academic background (as done by Sharma et al. [50]).

High school background was known for $74 \%$ and $76 \%$ of the Workshop Tutorial students and Map Meeters respectively, and obtained with informed consent. These fractions are large enough to capture the diversity of the class. The UAI is a ranking, so the nonparametric MannWhitney U test was used to compare the two groups; these were not statistically significantly different $(U=4411$; Workshop Tutorials: median $=91.80, N=112$; Map Meetings: median $=92.25, N=81 ; p=0.74$ ).

\section{Tutorial attendance}

Figure 3 shows the weekly attendance at the different tutorial types throughout the semester. The attendance at Map Meetings remained essentially constant, whereas Workshop Tutorials experienced a steady decline, with only $67 \%$ of initial attendance in the last week. These findings suggest that students found Map Meetings more worthwhile to attend than Workshop Tutorials.

Figure 4 shows how many students attended a given number of tutorials. The local context was such that students were given the freedom to swap tutorials.
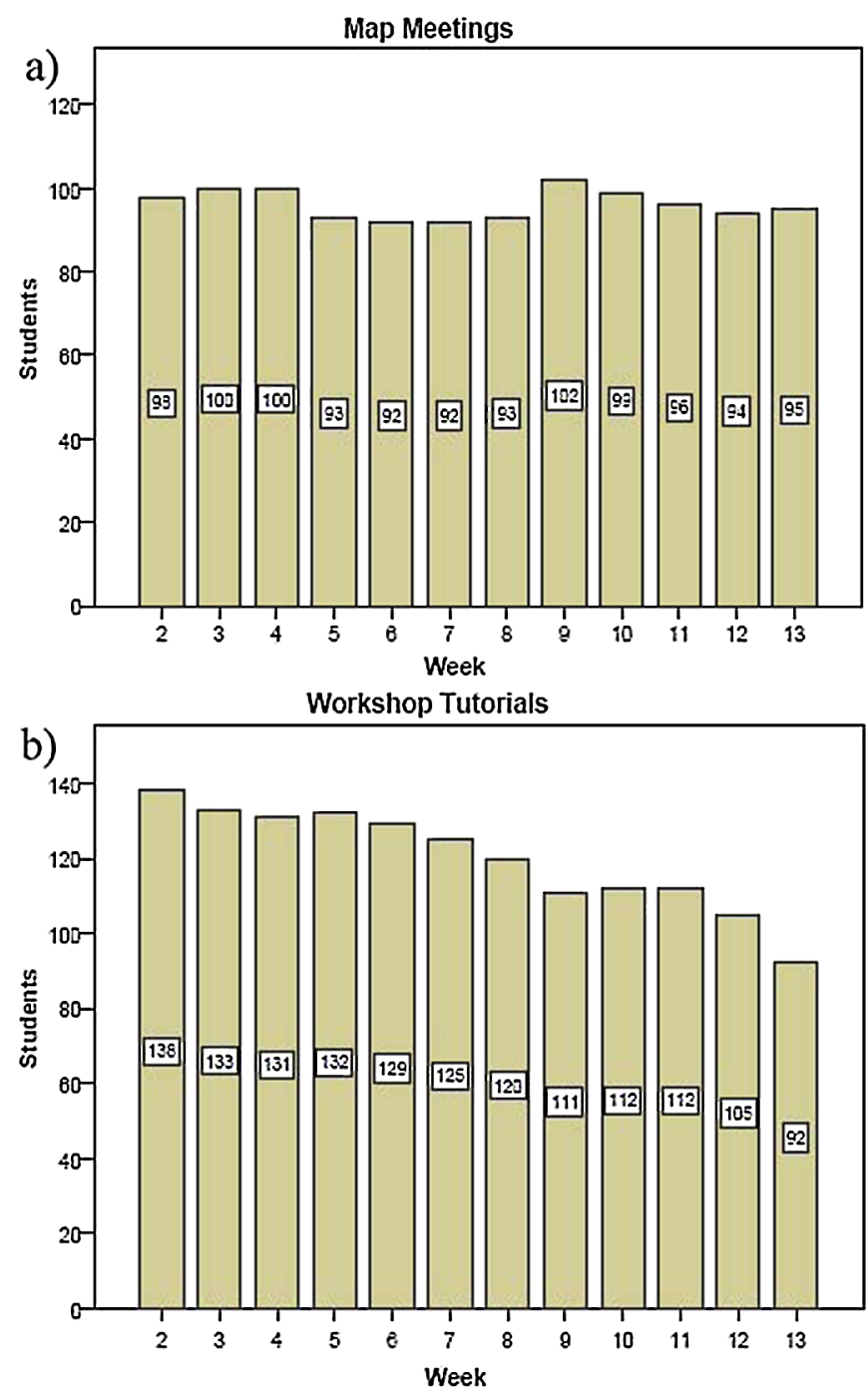

FIG. 3 (color online). Weekly attendance at Map Meetings (a) and Workshop Tutorials (b) in first semester. Tutorials start in week 2 .

Most students attended most tutorials. Students who attended at least ten tutorials were granted the $2 \%$ attendance mark. These students were called "persistent" as they had been committed to attending tutorials and had been "fully exposed" to their effect. Eighty percent of students who initially attended Map Meetings became persistent Map Meeters, whereas the equivalent fraction for Workshop Tutorials was $66 \%$. These findings support the earlier suggestion that Map Meetings was more popular with the students than the Workshop Tutorials.

Only 17 of all students attended at least one tutorial of each type during the semester. Of these, nine students changed from Workshop Tutorials to Map Meetings between weeks 4 and 9. Individual data on these students were studied in detail, and the only trend found was that the students were not the strongest in the class as measured by their UAI. Still, these were diligent students who attended 

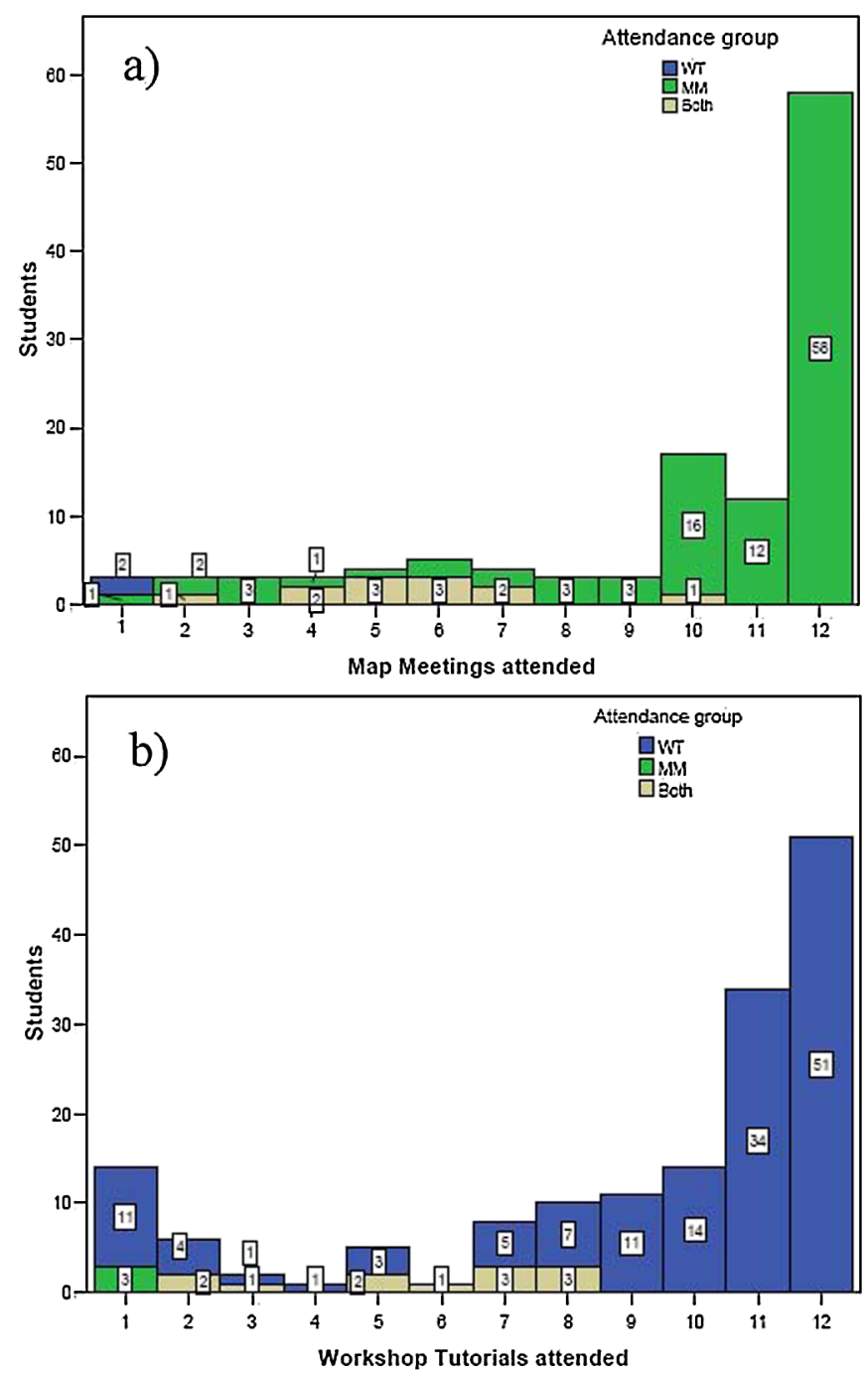

FIG. 4 (color online). Number of Map Meetings (MM) (a) and Workshop Tutorials (WT) (b) attended by students in first semester. With respect to the attendance groups, WT students attended at most one Map Meeting, MM students attended at most one Workshop Tutorial, and the "Both" students attended at least two of each type of tutorial.

between nine and 13 tutorials each. Only one of these students, however, was registered as a persistent student (in Map Meetings), as this required attendance at minimum ten tutorials of one tutorial type. No students swapped from Map Meetings to Workshop Tutorials.

The characteristics of persistent versus nonpersistent students will not be discussed due to the small number of nonpersistent students (particularly in Map Meetings) and the lack of homogeneity in attendances (ranging from one to nine tutorials).

\section{Qualitative analyses}

\section{Tutorial observations}

Observations of both types of tutorials were undertaken in week 9 by a physics education research expert not associated with the project. The observer was asked to comment on the level of involvement of tutors and students, and provide an overall impression of each tutorial.

With respect to the supervisors and tutors, the observer reported comparable levels of activity in both tutorials, all tutors being active most of the time. He did not observe any differences with respect to engagement or competency, and explicitly stated that in the Workshop Tutorial "[a]ll three tutors appear to be well-prepared and appreciative of techniques for promoting effective learning." In both tutorials it is noted that the tutors did more talking than listening. The only clear difference was the higher tutor-to-student ratio in the Workshop Tutorial.

In terms of the students, the observer reported no particular difference between the tutorials. He noted similar durations of on-task behavior, and a great variety of group behavior styles in both tutorials. However, there was a higher level of student-student interaction in the Workshop Tutorial than in the Map Meeting. During the summary lecture in the latter, about $20 \%$ of the students seemed to be "switched off," and the observer also commented that he "did not notice any group making explicit use of [the Link Map]."

Overall, two key differences between the two tutorial types were noted by the observer. First, the Map Meeting was more teacher centered (the observer commented that he "was a little surprised at the amount of didactic instruction"), whereas the Workshop Tutorial was more student centered. Second, the Workshop Tutorial had a more "casual atmosphere" than the structured Map Meeting.

\section{Focus group discussion}

Midway through second semester, students were invited to participate in focus groups to discuss their tutorial experience. Fourteen students from three different courses who all had knowledge of the two tutorial types volunteered. Regardless of course choice or high school physics background, all focus groups had very similar comments about the tutorials. This section, however, will only discuss the focus group discussion that involved the students who had attended the particular set of tutorials described in this paper.

Three students attended the focus group; two of these (one male, student A, and one female, student D) had attended Map Meetings in the first semester, whereas the last student (a female, student B) had attended Workshop Tutorials. In second semester all students attended Workshop Tutorials. The student with no experience with Map Meetings was still familiar with the Map Meeting materials. Students were asked to comment on the individual aspects of Map Meetings and compare the two types of tutorials. The focus group lasted for $32 \mathrm{~min}$ and was conducted by the first author (labeled "C"). The interview was transcribed and analyzed using thematic analysis [62]. 
All three students said that they would prefer to attend Map Meetings, given a choice. The following quote illustrates how students felt a greater sense of purpose in Map Meetings.

C: What would you say is the main difference, the most important difference between the two types of tutorials?

A: You know what you have to do in the Map Meetings, and you don't really know what you have to do in the [Workshop Tutorials] (...) Well, you know you have to answer the questions, but you don't know what to use and how to do it, and the concepts behind it.

B: Yeah, you don't know why you're doing it.

A clear theme to emerge from the focus group was that the students saw how all the different aspects of Map Meetings were directed towards preparing for the examination, and this encouraged them to stay on task. When discussing the tutorial problems, student A said about the tutorial problems, "[I]f you say that they're based on past exam questions, most kids would work. Because they'd want to know how to do the exam questions, in the tute."

Students claimed that they spent more time working in Map Meetings than in Workshop Tutorials, and felt that the Workshop Tutorial problems either were too difficult or not relevant.

In Map Meetings, students were particularly positive towards the Link Maps and the problem sheets. The layouts of both were motivating: students liked the colors and different features in the Link Maps, and the large font and handwritten style of the problem sheets. With respect to the Map Meeting problem sheets, student A said, "I find it's good because you know that they've thought about the question because they've had to hand write it, whereas [in Workshop Tutorials] they could've just copied it out of a textbook without thinking that it's gonna be in the exam or these are the concepts you need to know."

Student A said that the formulas on the map were extremely useful during the problem-solving session. The lack of a formula sheet in Workshop Tutorials was offered as a reason for why students often stopped working. Although the provision of formulas may appear to remove the necessity to think and understand the material, student A claims this was not the case.

"There's, like, at least one question for each of them [the formulas] on the sheet, so you've just got to work out which formula goes where. (...) I know it sounds bad, like, just putting a formula into a question, but also it helps you really understand the concept behind the formula, it's not just putting the formula in. Because you just know where the formula came from, which helps you understand the concept of that question."

Students found the maps a useful reference when doing their online assessments as well, suggesting transferability of the Link Maps for use in understanding physics.
Other issues that were mentioned were the solution sheets, the group work, the demonstrations, and the tutors. Students found the solution sheets in both types of tutorials useful. They liked how they could understand the question by reading the solutions if they had not initially understood the problem. There were mixed opinions about the group work: student A thought it was useful, whereas students B and $\mathrm{D}$ found that they learned less because they ended up talking about other things.

B: I find it easier to just work through it myself and just do it at my own pace. Whereas with the group I just talk to them about other stuff.

Lastly, in terms of the tutors in Workshop Tutorials, students thought they were good, but felt that they didn't always appreciate the problems the students faced, in particular, with respect to mathematics knowledge.

A: They're really good, but they just need to come down to our level, maybe. Of understanding. (...) I'm not very mathematically minded, but people can just, like, look at a formula and work out, you know, why things are, like, where they are. Apart from me ... I need concepts, but that's because I haven't done physics in year 12 and stuff.

When asked how Map Meetings could be improved, the students did not have many suggestions. However, they did request more than two tutors in each tutorial, and suggested that in some cases it would be beneficial with even more detailed solutions.

\section{Short-answer responses in questionnaire}

A questionnaire that included two short-answer questions was handed out in tutorials in the last week of semester asking which tutorial type students preferred and what they liked and disliked about the tutorial they were attending when completing the questionnaire. In Map Meetings 74 students responded, whereas in Workshop Tutorials 34 responded. Not all students answered both questions.

Only eight students had attended both types of tutorials and could thus provide any informed feedback on the question regarding which tutorial type they preferred. Of these students, six preferred Map Meetings whereas the remaining two preferred Workshop Tutorials. The following students' explanations are representative of the first group.

"Definitely the Map Meeting, as it made physics make so much more sense than the Workshop Tutorial, as everything was explained really basically so that it was easily understood, which then helped in being able to apply it to the problems. Also, the Workshop Tutorial questions were often too hard, especially when you don't fully understand the concepts."

"Definitely Map Meetings as the concepts were discussed prior to the questions to remind you of the concepts. They presented the concepts in logical and really well-presented manner. They were very very helpful. The problem in the other tuts seem too difficult and the tutors 
quite often say 'this is a tough question, don't worry too much."”

However, one of the students who preferred Workshop Tutorials provided an important reminder that one size does not fit all.

“[I preferred] Workshop: mind maps aren't useful to me. I prefer working through questions, and identifying where my weaknesses are. (...) [I]t would be better to work through the problems and then build a mind map, rather than the reverse."

When asked what they liked or disliked about the tutorial they were attending when filling in the questionnaire, the vast majority of responses targeted what the students had liked. In Map Meetings, the 74 students who responded to the questionnaire primarily mentioned the Link Maps (47 times) and the summary lecture (27 times). Note that both of these are strongly scaffolding activities. No other tutorial feature was mentioned more than 10 times. In Workshop Tutorials, the best liked features by the 34 responding students were group work (mentioned 12 times), the tutors (8), and the problem sheets (7). The demonstrations received very little mention (4 positive and 2 negative across both tutorial types).

We had expected that the visual aspect of the Link Maps would be particularly beneficial to novices [63]. Some students even explicitly mentioned the visual aspect.

"I have found from my experience that these type of tutorial [Map Meetings] have helped me a lot as I am a visual learner and found the sheets very useful."

"I learn visually, excellent summary of all important ideas, formulas, concepts! Great studying tool! Simplified learning! Easy to understand!!"

In summary, the students appreciated the scaffolding Map Meeting environment and felt that this helped them understand difficult concepts and prepare them for solving problems. In fact, of the Workshop Tutorial students who had never attended Map Meetings, five mentioned that they would like more structure in the form of a summary lecture at the beginning and/or going through a problem on the board at the end, as illustrated by the following comments.

"It is good to be able to work in a group and solve problems together. But the tutorial needs a bit more structure, it would be better if the tutors revised lecture material with us and then worked through the problems."

"I prefer the demonstrators write something on the board first (e.g., review of lessons, what we should have learnt, formulas, etc., ...) before we start the tutorial."

"[P]erhaps some general explanations of an approach to answering the specific questions because sometimes it is difficult to know where to begin."

\section{Other qualitative feedback}

Other qualitative feedback regarding the tutorials came via Emails and the Student Staff Liaison Meeting at the end of the semester. In Emails (as well as verbally) Workshop Tutorial students asked if they could receive the material handed out in Map Meetings. All such requests were denied to try to keep the two tutorial groups as separate as possible. The extent of these requests is unknown, but physics students generally work together outside of class, in particular, in the residential colleges at the university, and there is a strong culture of sharing material amongst peers. Unfortunately, it is therefore uncertain how many Workshop Tutorial students acquired the Map Meeting material.

In the Student Staff Liaison Meeting at the end of the semester, two students from each course are invited to gather feedback from peers and present feedback on their course. In the Fundamentals course the feedback on tutorials was very positive towards Map Meetings, which were referred to as "fantastic."

"[The tutorial supervisor] clearly explains things prior to starting and revises essentials and runs through difficult problems at the end. I have found them better than the

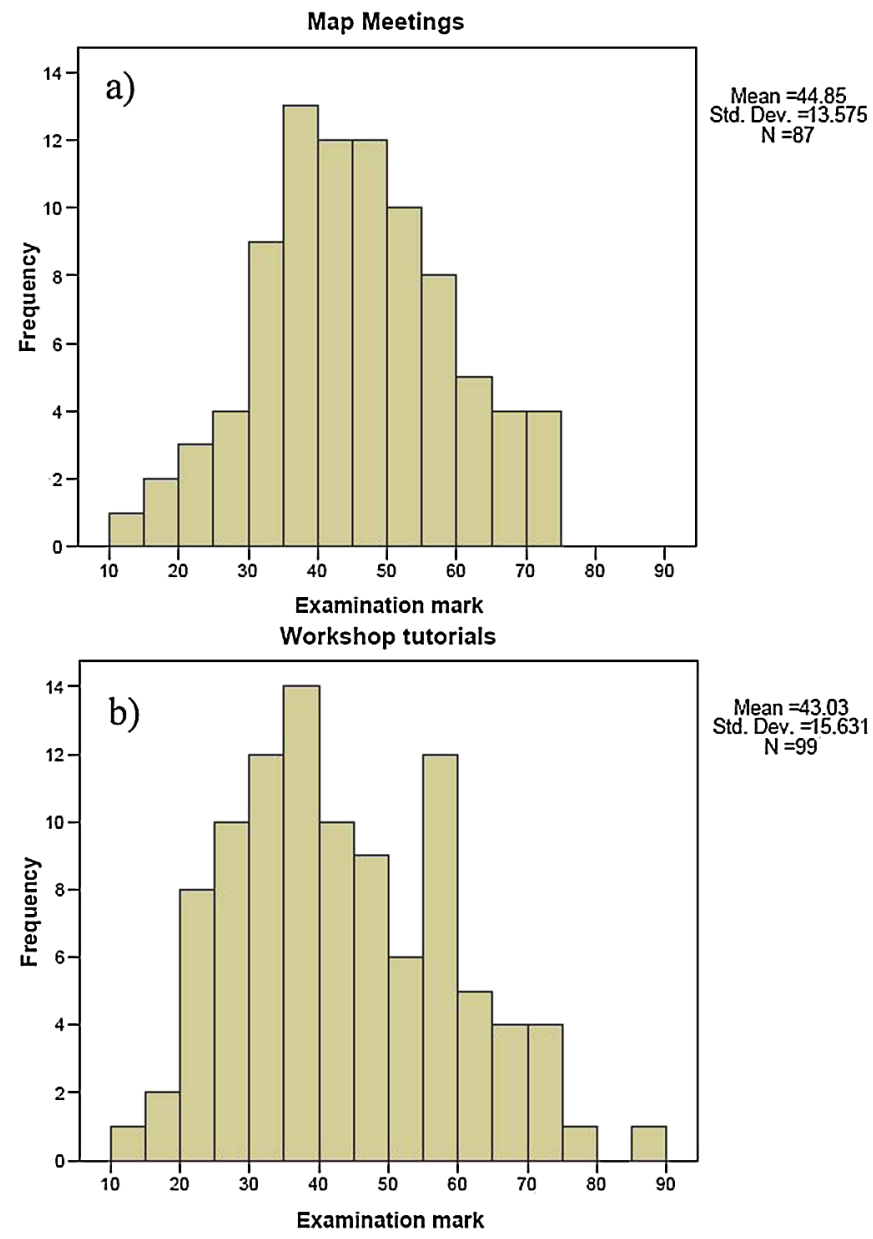

FIG. 5 (color online). Histograms of marks for all persistent students in Map Meetings (a) and Workshop Tutorials (b) in the end-of-semester examination. Note that each bin refers to the number of students who received from the lower mark inclusive to the upper mark exclusive. 
TABLE II. Percentage of persistent students receiving low, medium, and high marks, respectively, in the final examination.

\begin{tabular}{lccc}
\hline \hline Tutorial group & Low achieving $(<30$ marks $)$ & Medium achieving $(30-59$ marks $)$ & High achieving $(\geq 60$ marks $)$ \\
\hline Workshop Tutorials $(N=99)$ & $21 \%(N=21)$ & $64 \%(N=63)$ & $15 \%(N=15)$ \\
Map Meetings $(N=87)$ & $11 \%(N=10)$ & $74 \%(N=64)$ & $15 \%(N=13)$ \\
\hline \hline
\end{tabular}

lectures!! I can learn and remember so much more from them. And in comparison to the [Workshop Tutorials] they have been far more beneficial." [sic]

\section{E. Analysis of examination results}

The distributions of examination results were normal for all persistent students, so independent samples t-tests were performed to compare the means. There was no statistically significant difference between the tutorial groups $(t=0.842$; Workshop Tutorials: mean $=43.03$, $\mathrm{SD}=15.631, N=99 ;$ Map Meetings: mean $=44.85$, $\mathrm{SD}=13.575, N=87 ; p=0.401)$, but we note that there is a difference of 1.82 marks in favor of Map Meetings.

However, since the Map Meeting tutorials were specifically designed to give students a solid foundation in physics (rather than to stretch the highest achievers), we investigated the distributions of marks with respect to high, medium, and low achievers. The examination had a maximum mark of 90, so high achievers were defined as those obtaining at least 60 marks, medium achievers 30-59 marks, and low achievers less than 30 marks. Figure 5 shows the histograms of marks for the different tutorial groups, whereas Table II shows the percentage of students who fall into the low-, medium-, and high-achieving categories.

From Table II it appears that although the two tutorial types are equally successful at producing high-achieving students, there is a marked difference amongst the lowachieving students. Almost twice the fraction of Workshop Tutorial students is in the low-achieving region compared to the Map Meeters. Ultimately, for these students without prior formal physics instruction the distinction between low and medium achieving is much more important than the distinction between medium achieving and high achieving, as the former is related to failing or passing the course. Therefore, the results suggest that Map Meetings primarily makes a difference to the students who are finding physics more challenging.

\section{DISCUSSION}

Triangulating the results by considering them in concert, Map Meetings - representing the more scaffolding tutorial environment-was clearly the preferred tutorial type by physics novices. Map Meetings was preferred primarily because of the Link Maps and the summary lecture: students liked the structure these features brought to the learning environment, and several Workshop Tutorial students requested such scaffolding even though they had never attended a Map Meeting.

Why did students prefer the more strongly scaffolding Map Meeting tutorial environment? The main reason to emerge from the student feedback is the stronger sense of purpose the Map Meeting environment offered; students knew what they were doing and why. Overwhelmingly, students' focus and interest in the material centered around the final examination. This is only to be expected of novices who have only engaged with physics (at least in a formal sense) for a few months [11]. Personal interest develops with prior knowledge [11]; how can one be interested in something one knows hardly anything about? Also, in terms of strategic processing, the students have not developed their skills enough to confidently apply these without needing access to supportive experts.

The way the Map Meetings were scaffolding was well received by the students. This provides further support for using the theoretical foundation this work is based on. Students felt the summary lecture with the Link Map was clear and that the explanations made sense of the material, suggesting that students were not cognitively overloaded. The nonlinear presentation of material on the Link Maps seemed logical to the students, supporting the case for letting the structure of physics knowledge itself inform the layout.

The examination results suggested that the academically weakest students benefitted the most from Map Meetings. Map Meetings was successful in achieving its aim, which was to design a tutorial environment that would help students with no prior formal physics instruction gain a basic understanding of physics. For the strongest students, however, the type of tutorial attended did not appear to influence their performance. This finding is interesting because it provides some preliminary information about what level of scaffolding is optimal at different levels of student competency. As discussed by Alexander [11] and van Merrienboër and Sweller [15], the greater the competency, the lower the necessary scaffolding. However, no quantitative details were offered for this qualitative statement, for good reason, given that it is impossible to accurately quantify a person's level of domain knowledge. However, our study suggests that the level of competency for which a strongly scaffolding tutorial environment is more beneficial than a relatively minimally scaffolding one is quite low in a tertiary physics environment. At higher levels of competency, the two tutorial types appeared 
equally successful for learning. The competency level for which a minimally scaffolding environment is more beneficial seems to lie beyond the competency of the students covered in this study.

It is important to keep in mind, however, that this conclusion is based on the students who chose to attend at least ten tutorials, which is strongly related to how beneficial students perceived the tutorials to be. In this study, Map Meetings was more motivating than Workshop Tutorials. Hence, educators must consider the interplay between the educational benefit of various environments given that students engage with it, combined with the likelihood that students will engage with the environment. A pedagogically excellent tutorial is of no use if no students attend. However, it is important to realize that these two features are theoretically separate, and although it is the combination of the two that is relevant in practice, studying them independently will allow for evaluation of the features separately.

Finally, we discuss the strengths and limitations of the study. First, the lack of truly randomized treatment and control groups was unavoidable. The comparison of students' academic backgrounds in terms of UAI suggested that the groups were not statistically significantly different, but this cannot be guaranteed without true random sampling. On the other hand, the naturalistic setting is also a strength because the results represent how students act in a real and extended learning situation. After all, this is what environments such as Map Meetings are designed for. In an artificial and contrived laboratory experiment, the students are not as personally invested as their performance generally is of no consequence to them. Another limitation is the lack of clear boundary between the two tutorial environments. Owing to the naturalistic nature of the study, it was impossible to guarantee that students in Workshop Tutorials did not acquire Map Meeting material, and that Map Meeting students did not get the Workshop Tutorial sheets. This potentially reduces the difference in examination marks if a considerable fraction of the Workshop Tutorial students used Link Maps in their examination preparation. A third limitation is the potential confounding factor of a "teacher effect" [64], i.e., that the presence of the first author in the Map Meetings (but not Workshop Tutorials) may have affected the results of the study. Although this cannot be ruled out, we point out that in their feedback, students primarily referred to the tutorial structure-rather than the tutorial supervisor-when highlighting which aspects of the tutorial they liked, and that the external observer did not note any difference between the enthusiasm of tutors in the two different types of tutorials.

\section{CONCLUSION}

Reviewing the findings in light of the original research questions, we first note that there were clear patterns in the student attendance in both tutorial types: in Map Meetings attendance remained essentially constant, whereas Workshop Tutorials experienced a steady decline to $67 \%$ of original attendance by the end of the semester.

Both tutorial types were observed by an expert in physics education. He commented that the main differences between the tutorials were the higher degree of scaffolding in Map Meetings and a more casual atmosphere in Workshop Tutorials. There were no differences in tutor activity, engagement, or competency or in student on-task behavior, except for a higher level of studentstudent interaction in Workshop Tutorials.

In terms of student experiences, students were clearly in favor of Map Meetings. Focus group discussions revealed that students felt a greater sense of purpose in Map Meetings, that the Link Maps were very helpful, and that there was a greater appreciation for the problems the students faced as compared to in Workshop Tutorials. Feedback on short-answer questionnaires mirrored this; in particular, the students favorably mentioned the Link Maps and summary lectures-both strongly scaffolding activities.

Analysis of the end-of-semester examination showed that Workshop Tutorials had nearly twice the fraction of low-achieving students (21\%) compared to Map Meetings $(11 \%)$, clearly indicating that Map Meetings made a difference to those who were most challenged by the physics course and thus most at risk of failing.

Hence, Map Meetings emerges as the more beneficial tutorial environment for students without a high school physics background when compared to Workshop Tutorials, which - and this should not be underestimatedare already established as a valuable learning environment.

\section{IMPLICATIONS FOR LEARNING AND TEACHING, AND FURTHER WORK}

The implication for learning and teaching is first and foremost that one size does not fit all: educators must carefully consider the level of competency of their students when choosing learning environments. A corollary is that the range of competencies in one class should ideally not be so large as to span groups who would benefit from different types of learning environments; i.e., this study supports streaming of students with respect to prior knowledge (as is done at the University of Sydney).

This study also adds to the debate on student-centered versus teacher-centered learning by highlighting that not only is this debate far from black and white, the shades of gray vary themselves as other variables (such as prior knowledge and experience) are considered.

Further work of great interest would be to extend the study to include students of higher levels of competency and investigate whether the trend of reduced need for scaffolding for higher prior knowledge can be detected. It would also be worthwhile pursuing an experiment in which 
the treatment and control groups have no interaction whatsoever. Lastly, an aspect that was not investigated here, but which may reveal interesting results, is to measure how much time students spent on their physics studies. If more strongly scaffolding environments allow students to reach a certain level of competency in a shorter period of time, this is a benefit that cannot be measured by examination performance alone.

\section{ACKNOWLEDGMENTS}

The authors would like to thank the Sydney University Physics Education Group for valuable discussions and comments of drafts of the paper, as well as the anonymous referees who provided extensive feedback which significantly helped to improve the paper.
[1] B. Acar and L. Tarhan, Effects of cooperative learning on students' understanding of metallic bonding, Res. Sci. Educ. 38, 401 (2007).

[2] P. A. Kirschner, J. Sweller, and R. E. Clark, Why minimal guidance during instruction does not work: An analysis of the failure of constructivist, discovery, problem-based, experiential, and inquiry-based teaching, Educ. Psychol. 41, 75 (2006).

[3] Wahyudi and D.F. Treagust, An investigation of science teaching practices in Indonesian rural secondary schools, Res. Sci. Educ. 34, 455 (2004).

[4] H. G. Schmidt et al., Problem-based learning is compatible with human cognitive architecture: Commentary on Kirschner, Sweller, and Clark, Educ. Psychol. 42, 91 (2007).

[5] C.E. Hmelo-Silver, R. G. Duncan, and C.A. Chinn, Scaffolding and achievement in problem-based and inquiry learning: A response to Kirschner, Sweller, and Clark , Educ. Psychol. 42, 99 (2007).

[6] P. Fensham, J. Garrard, and L. West, The use of cognitive mapping in teaching and learning strategies, Res. Sci. Educ. 11, 121 (1981).

[7] J. Sweller, P. A. Kirschner, and R. E. Clark, Why minimally guided teaching techniques do not work: A reply to commentaries, Educ. Psychol. 42, 115 (2007).

[8] T.J. Bing and E.F. Redish, Analyzing problem solving using math in physics: Epistemological framing via warrants, Phys. Rev. ST Phys. Educ. Res. 5, 020108 (2009).

[9] J. Tuminaro and E.F. Redish, Elements of a cognitive model of physics problem solving: Epistemic games, Phys. Rev. ST Phys. Educ. Res. 3, 020101 (2007).

[10] M. Crotty, The Foundations of Social Research (Allen \& Unwin, Sydney, 1998).

[11] P. A. Alexander, The development of expertise: The journey from acclimation to proficiency, Educ. Researcher 32, 10 (2003).

[12] I. Abrahams and R. Millar, Does practical work really work? A study of the effectiveness of practical work as a teaching and learning method in school science, Int. J. Sci. Educ. 30, 1945 (2008).

[13] M. Cook, G. Carter, and E. N. Wiebe, The interpretation of cellular transport graphics by students with low and high prior knowledge, Int. J. Sci. Educ. 30, 239 (2008).

[14] H. Sturm and F.X. Bogner, Student-oriented versus teacher-centred: The effect of learning at workstations about birds and bird flight on cognitive achievement and motivation, Int. J. Sci. Educ. 30, 941 (2008).

[15] J. J. G. van Merrienboër and J. Sweller, Cognitive load theory and complex learning: Recent developments and future directions, Educ. Psychol. Rev. 17, 147 (2005).

[16] C. A. Stone, The metaphor of scaffolding: Its utility for the field of learning disabilities, J. Learn. Disabil. 31, 344 (1998).

[17] M. Pressley et al., Some of the reasons why preparing for exams is so hard: What can be done to make it easier?, Educ. Psychol. Rev. 9, 1 (1997).

[18] C. Reigosa and M.-P. Jiminez-Aleixandre, Scaffolded problem-solving in the physics and chemistry laboratory: Difficulties hindering students' assumption of responsibility, Int. J. Sci. Educ. 29, 307 (2007).

[19] J. A. Leon and O. Perez, The influence of prior knowledge on the time course of clinical diagnosis inferences: A comparison of experts and novices, Discourse Process. 31, 187 (2001).

[20] P.P. Afflerbach, The influence of prior knowledge on expert readers' main idea construction strategies, Read. Res. Q. 25, 31 (1990).

[21] M. Siew Lian, An investigation into high-achiever and low-achiever knowledge organisation and knowledge processing in concept mapping: A case study, Res. Sci. Educ. 28, 337 (1998).

[22] A. Ben-David and A. Zohar, Contribution of metastrategic knowledge to scientific inquiry learning, Int. J. Sci. Educ. 31, 1657 (2009).

[23] M. W. Eysenck and M. T. Keane, Cognitive Psychology: A Student's Handbook (Psychology Press, Hove, England, 2005), 5th ed.

[24] E.F. Redish, in A Theoretical Framework for Physics Education Research: Modeling student thinking, Proceedings of the International School of Physics "Enrico Fermi," Course CLVI, edited by E. F. Redish and M. Vicentini (IOS Press, Amsterdam, 2004).

[25] J. Sweller, J. J. G. van Merriënboer, and F. G. W. C. Paas, Cognitive architecture and instructional design, Educ. Psychol. Rev. 10, 251 (1998).

[26] M.F. Bear, B.W. Connors, and M. A. Paradiso, Neuroscience: Exploring the Brain (Lippincott Williams \& Wilkins, Philadelphia, 2007).

[27] J. D. Novak, Learning, Creating, and Using Knowledge; Concept Maps as Facilitative Tools in Schools and 
Corporations (Lawrence Erlbaum Associates, Mahwah, NJ, 1998).

[28] O. Kaya, A student-centred approach: Assessing the changes in prospective science teachers' conceptual understanding by concept mapping in a general chemistry laboratory, Res. Sci. Educ. 38, 91 (2007).

[29] M. E. Patterson, D. F. Dansereau, and D. Newbern, Effects of communication aids and strategies on cooperative teaching, J. Educ. Psychol. 84, 453 (1992).

[30] K.L. Rewey et al., Effects of scripted cooperation and knowledge maps on the processing of technical material, J. Educ. Psychol. 81, 604 (1989).

[31] G. B. Kilic, Concept maps and language: A Turkish experience, Int. J. Sci. Educ. 25, 1299 (2003).

[32] C. Buntting, R. K. Coll, and A. Campbell, Student views of concept mapping use in introductory tertiary biology classes, Int. J. Sci. Math. Educ. 4, 641 (2005).

[33] J. A. Heinze-Fry and J. D. Novak, Concept mapping brings long-term movement toward meaningful learning, Sci. Educ. 74, 461 (1990).

[34] F. N. Keraro, S. W. Wachanga, and W. Orora, Effects of cooperative concept mapping teaching approach on secondary school students' motivation in biology in Gucha district, Kenya. Int. J. Sci. Math. Educ. 5, 111 (2006).

[35] L. Brandt et al., The impact of concept mapping and visualization on the learning of secondary school chemistry students, Int. J. Sci. Educ. 23, 1303 (2001).

[36] P. G. Markow and R. A. Lonning, Usefulness of concept maps in college chemistry laboratories: Students' perceptions and effects on achievement, J. Res. Sci. Teach. 35, 1015 (1998).

[37] W. J. Pankratius, Building an organized knowledge base: Concept mapping and achievement in secondary school physics, J. Res. Sci. Teach. 27, 315 (1990).

[38] W.-M. Roth and A. Roychoudhury, The concept map as a tool for the collaborative construction of knowledge: A microanalysis of high school physics students, J. Res. Sci. Teach. 30, 503 (1993).

[39] S. K. Ingec, Analysing concept maps as an assessment tool in teaching physics and comparison with the achievement tests, Int. J. Sci. Educ. 31, 1897 (2009).

[40] C. Lindstrøm and M.D. Sharma, Link maps and map meetings: Scaffolding student learning, Phys. Rev. ST Phys. Educ. Res. 5, 010102 (2009).

[41] M.D. Sharma et al., Learning outcomes and curriculum development in physics: a report on tertiary physics teaching and learning in Australia commissioned by the Australian Universities Teaching Committee, 2005, http://www.physics.usyd.edu.au/super/AUTC/.

[42] C.E. Wieman and K. K. Perkins, Transforming physics education, Phys. Today 58, No. 11, 36 (2005).

[43] P. Ramsden, Learning to Teach in Higher Education (RoutledgeFalmer, London, 2003).

[44] R. Hake, Interactive-engagement versus traditional methods: A six-thousand-student survey of mechanics test data for introductory physics courses, Am. J. Phys. 66, 64 (1998).

[45] K. Cummings et al., Evaluating innovation in studio physics, Am. J. Phys. 67, S38 (1999).

[46] C. M. Sorensen et al., The new studio format for instruction of introductory physics, Am. J. Phys. 74, 1077 (2006).
[47] C. Henderson and M.H. Dancy, Barriers to the use of research-based instructional strategies: The influence of both individual and situational characteristics, Phys. Rev. ST Phys. Educ. Res. 3, 020102 (2007).

[48] W. Damon and E. Phelps, Critical distinctions among three approaches to peer education, Int. J. Educ. Res. 13, 9 (1989).

[49] K. M. Koenig, R. J. Endorf, and G. A. Braun, Effectiveness of different tutorial recitation teaching methods and its implications for TA training, Phys. Rev. ST Phys. Educ. Res. 3, 010104 (2007).

[50] M. D. Sharma, R. Millar, and S. Seth, Workshop Tutorials: Accommodating student-centred learning in large first year university physics courses, Int. J. Sci. Educ. 21, 839 (1999).

[51] M. D. Sharma, A. Mendez, and J. W. O'Byrne, The relationship between attendance in student-centred physics tutorials and performance in university examinations, Int. J. Sci. Educ. 27, 1375 (2005).

[52] E. R. Kandel, I. Kupfermann, and S. Iversen, in Principles of Neural Science, edited by E. R. Kandel, J. H. Schwartz, and T.M. Jessell (McGraw-Hill, New York, 2000), p. 1228.

[53] C. W. Eriksen and J. D. St. James, Visual attention within and around the field of focal attention: A zoom lens model, Percept. Psychophys. 40, 225 (1986).

[54] L. S. Vygotsky, in Mind in Society: The Development of Higher Psychological Processes, edited by M. Cole, V. John-Steiner, S. Scribner, and E. Souberman (Harvard University Press, Cambridge, MA, 1978).

[55] N. Webb, Task-related verbal interaction and mathematics learning in small groups, J. Res. Math. Educ. 22, 366 (1991).

[56] D. L. Schwartz and J.D. Bransford, A time for telling, Cogn. Instr. 16, 475 (1998).

[57] E. Gaigher, J. M. Rogan, and M. W. H. Braun, Exploring the development of conceptual understanding through structured problem-solving in physics, Int. J. Sci. Educ. 29, 1089 (2007).

[58] K. L. Malone, Correlations among knowledge structures, force concept inventory, and problem-solving behaviors, Phys. Rev. ST Phys. Educ. Res. 4, 020107 (2008).

[59] H. M. Johnston et al., The research-teaching nexus in physics: Scholarship into teaching and learning, Aust. J. Phys. 44, 66 (2007).

[60] M.D. Sharma et al., An investigation of the effectiveness of electronic classroom communication systems in large lecture classes, Australas. J. Educ. Tech. 21, 137 (2005).

[61] The Handbook of Qualitative Research, edited by N. K. Denzin and Y.S. Lincoln (Sage Publications, Thousand Oaks, CA, 2000).

[62] G. W. Ryan and H. R. Bernard, in Handbook of Qualitative Research, edited by N. K. Denzin and Y. S. Lincoln (Sage Publications, Thousand Oaks, CA, 2000), p. 769.

[63] R. M. Felder, Reaching the second tier: Learning and teaching styles in college science education, J. Coll. Sci. Teach. 22, 286 (1993).

[64] B. Nye, S. Konstantopoulos, and L. V. Hedges, How large are teacher effects? , Educ. Eval. Policy Anal. 26, 237 (2004). 